\title{
Prognostic significance of circulating laminin gamma2 for early-stage non-small-cell lung cancer
}

This article was published in the following Dove Press journal:

OncoTargets and Therapy

7 July 2016

Number of times this article has been viewed

\author{
Yu Teng ${ }^{1, *}$ \\ Zitong Wang ${ }^{2, *}$ \\ Li Mal,* \\ Lina Zhang' \\ Yinan Guo' \\ Meng Gu' \\ Ziyu Wang' \\ Yue Wang' \\ Wentao Yue' \\ 'Department of Cellular and \\ Molecular Biology, Beijing Chest \\ Hospital, Capital Medical University, \\ Beijing Tuberculosis and Thoracic \\ Tumor Research Institute, Beijing, \\ People's Republic of China; \\ ${ }^{2}$ Department of Thoracic Surgery, \\ Beijing Chest Hospital, Capital \\ Medical University, Beijing, People's \\ Republic of China \\ *These authors contributed equally \\ to this work
}

\begin{abstract}
Background: Laminin gamma2 ( $\operatorname{Ln}-\gamma 2)$ chain, a distinctive subunit of heterotrimeric laminin-332, is frequently upregulated in carcinomas and is of great importance in cell migration and invasion. Despite this, the status of circulating Ln- $\gamma 2$ in lung cancer patients is still uncertain.

Patients and methods: In this retrospective study, serum samples from 538 all-stage (stages I-IV) patients with non-small-cell lung cancer (NSCLC) and 94 age-matched healthy volunteers were investigated by enzyme-linked immunosorbent assay. Data were statistically analyzed in combination with clinicopathological information.

Results: Circulating Ln- $\gamma 2$ was markedly increased in NSCLC, even in stage I cases $(P<0.01)$, reflecting the progression of lung cancer. Survival analysis on 370 eligible patients indicated that serum Ln- $\gamma 2$-negative patients survived much longer compared with Ln- $\gamma 2$-positive individuals $(P=0.028)$, and it was especially the case for stage I $(P<0.001)$, stage T1 $(P=0.001)$, and stage N0 patients $(P=0.038)$, all of whom represented early-stage cases. For the advanced patients, however, overall survivals were not significantly different among stages II-IV $(P=0.830)$, stages T2-T4 ( $P=0.575)$, stages N1-N3 ( $P=0.669)$, and stage M1 $(P=0.849)$. Cox analysis subsequently defined serum Ln- $\gamma 2$ as an independent prognostic indicator of NSCLC, particularly for earlystage patients. Furthermore, we demonstrated the association of serum Ln- $\gamma 2$ with smoking behavior, but its association with tumor progression and early prognostic significance were not altered in the nonsmoking cohort.

Conclusion: Our study demonstrated that elevation of circulating Ln- $\gamma 2$ was an early-emerging event in NSCLC and was significantly associated with poor prognosis in NSCLC, especially for early-stage cases.
\end{abstract}

Keywords: laminin gamma2, circulation, prognosis, early stage, non-small-cell lung cancer

\section{Introduction}

Among the various malignancies, lung cancer has long been considered to be the most common cause of cancer death. Non-small-cell lung cancer (NSCLC), which accounts for $\sim 80 \%$ of all lung carcinomas, progresses rapidly with a generally poor 5-year survival ranging from $6 \%$ to $18 \% .^{1}$ Most of these patients die of the late occurrence of multiorgan metastases. In fact, prior to clinical manifestations, metastatic dissemination mostly requires sequential cellular events, including local invasion, intravasation to the vessels, survival in the circulation, extravasation, and colonization. ${ }^{2}$

Laminins are a family of heterotrimeric extracellular glycoproteins functioning in all basement membranes (BMs). Thus far, at least 17 laminin isoforms have been identified, ${ }^{3}$ each with a cross-shaped and specific combination of $\alpha, \beta$, and $\gamma$ subunits. Laminin-332 (Ln-332), formerly termed laminin-5 by order of discovery, consists of heterogeneous $\alpha 3, \beta 3$, and $\gamma 2$ chains and serves as an essential structural constituent of BMs. It is certain that Ln-332 plays crucial roles in cell adhesion and migration. ${ }^{4}$ Junctional epidermolysis
Correspondence: Wentao Yue Department of Cellular and Molecular Biology, Beijing Chest Hospital, Capital Medical University, Beijing Tuberculosis and Thoracic Tumor Research Institute, 97 Machang, Beijing 10I 149, People's Republic of China

Tel +86 I0 89509372

Fax +86 I0 80507349

Email yuewt@ccmu.edu.cn BY
hereby accept the Terms. Non-commercial uses of the work are permitted without any furcher permission from Dove Medical Press Limited, provided the work is properly attributed. For permission for commercial use of this work, please see paragraphs 4.2 and 5 of our Terms (https://www.dovepress.com/terms.php). 
bullosa, a group of autosomal recessive skin disorders, manifests as mechanically induced blistering of fragile skin and mucosa. In the context of the etiology, it is basically caused by mutations in the coding gene of any subunit of the heterotrimer. ${ }^{5}$ Laminin functions as a bridge between the epidermis and the underlying connective tissues mainly through interaction with integrin receptors in some cell matrix adhesive structures, such as hemidesmosomes. Moreover, accumulating studies report that Ln-332 is frequently upregulated in some types of malignancies, ${ }^{6,7}$ especially in invading cancer cells or at the invasive tumor front. ${ }^{8,9}$ Intriguingly, under the interfacial tumor microenvironment, invading cancer cells could confer anoikis-resistant cell viability on surrounding myofibroblasts via upregulation of Ln-332 and its integrin receptors during invasion-associated tissue remodeling. ${ }^{10}$

Laminin gamma2 ( $\mathrm{Ln}-\gamma 2)$ chain, which is unique to the Ln-332 trimer, can be secreted out as a monomer ${ }^{11}$ and contributes to illustrating the characterization of cell motility. ${ }^{12,13}$ As previously reported, secretion of monomeric Ln- $\gamma 2$ was strongly induced in the presence of some regulatory cytokines in carcinoma cells, and this consequently promoted in vitro cell migration and in vivo aggressive tumor growth. ${ }^{14,15}$ Histochemical data repeatedly demonstrated that Ln- $\gamma 2$ staining was in close relation to the aggressive stages of tumor progression $^{16,17}$ and might be of great significance for the prognosis of varied carcinomas, ${ }^{18,19}$ including lung cancer. ${ }^{20}$ In a histological cohort study of colorectal polyps, generally recognized as precancerous lesions of colorectal carcinomas, Ln- $\gamma 2$ staining was claimed to be negative in all hyperplastic sections, whereas it tended to increase progressively toward more atypical phenotypes, suggesting its potential indication for early tumor invasiveness. ${ }^{21}$ Another investigation stated that overexpressed Ln- $\gamma 2$ was closely associated with tumor budding or locoregional failure at the invasive front of $\mathrm{T} 1$ colorectal cancers, which underpins it as a novel indicator for incipient tumor aggressiveness of small-sized colorectal carcinomas. ${ }^{22}$ Additionally, findings based on 102 small-sized lung carcinomas revealed that Ln- $\gamma 2$ was positive in most early-stage tumor tissues, implying a more valuable significance in the early diagnosis of lung cancer. ${ }^{9}$ In recent years, however, serologic approaches are becoming increasingly available in clinical investigations due to their rapid and convenient sampling method. Until now, only a few studies on circulating Ln- $\gamma 2$ have shown its prognostic potential for certain cancer types. ${ }^{23-25}$ Nevertheless, little is known about the significance of serum Ln- $\gamma 2$ in the early prognosis of tumor patients, especially in the field of lung cancer.

Herein, we performed a case-control study on the circulating levels of Ln- $\gamma 2$ in a cohort of 538 NSCLC patients scattered across all clinical stages and in 94 age-matched healthy volunteers. The associations between circulating Ln- $\gamma 2$ and lung cancer diagnosis and prognosis were primarily evaluated, especially in early-stage NSCLC individuals.

\section{Patients and methods \\ Patients and samples}

Between January 2008 and December 2011, serum samples from 538 cases of primary NSCLC patients and 94 agematched healthy volunteers were consecutively collected by the staff of Beijing Chest Hospital Sample Bank (Beijing, People's Republic of China). The detailed clinical data refined from the medical records are presented in Table 1. For survival analysis, 370 eligible patients who underwent a follow-up visit

Table I Associations between clinicopathological characteristics and serum Ln- $\gamma 2$ status in 538 NSCLC patients

\begin{tabular}{|c|c|c|c|c|}
\hline Variables & $\begin{array}{l}\text { No of } \\
\text { cases }\end{array}$ & Ln- $\gamma 2$ positive & Ln- $\gamma 2$ negative & $P$-value \\
\hline Total cases & 538 & 201 (37.4\%) & 337 (62.6\%) & \\
\hline Age (years) & & & & 0.650 \\
\hline$\leq 60$ & 293 & 112 (38.2\%) & $181(61.8 \%)$ & \\
\hline$>60$ & 245 & $89(36.3 \%)$ & 156 (63.7\%) & \\
\hline Sex & & & & $<0.00 I^{*}$ \\
\hline Male & 370 & I6I (43.5\%) & 209 (56.5\%) & \\
\hline Female & 168 & $40(23.8 \%)$ & 128 (76.2\%) & \\
\hline Smoking history & & & & $<0.00 I^{*}$ \\
\hline Smoker & 325 & I 46 (44.9\%) & $179(55.1 \%)$ & \\
\hline Nonsmoker & 213 & $56(26.3 \%)$ & I 57 (73.7\%) & \\
\hline Pathologic type & & & & 0.879 \\
\hline$S$ & 211 & $78(37.0 \%)$ & 133 (63.0\%) & \\
\hline$A$ & 327 & $123(37.6 \%)$ & 204 (62.4\%) & \\
\hline Differentiation & & & & 0.717 \\
\hline Poor & 219 & $79(36.1 \%)$ & 140 (63.9\%) & \\
\hline Moderate & 280 & $93(33.2 \%)$ & $187(66.8 \%)$ & \\
\hline Well & 39 & $12(30.8 \%)$ & 27 (69.2\%) & \\
\hline PT status & & & & $<0.00 I^{*}$ \\
\hline PTI & 89 & $20(22.5 \%)$ & 69 (77.5\%) & \\
\hline PT2 & 232 & 75 (32.3\%) & $157(67.7 \%)$ & \\
\hline PT3 & 43 & $23(53.5 \%)$ & 20 (46.5\%) & \\
\hline pT4 & 174 & $80(46.0 \%)$ & 94 (54.0\%) & \\
\hline $\mathrm{pN}$ status & & & & 0.154 \\
\hline pNO & 198 & $62(31.3 \%)$ & 136 (68.7\%) & \\
\hline $\mathrm{pNI}$ & 38 & 17 (44.8\%) & 21 (55.2\%) & \\
\hline $\mathrm{pN} 2$ & 192 & $71(37.0 \%)$ & 121 (63.0\%) & \\
\hline $\mathrm{pN} 3$ & 110 & 47 (42.7\%) & 63 (57.3\%) & \\
\hline pM status & & & & $0.001 *$ \\
\hline pMO & 312 & 98 (31.4\%) & $214(68.6 \%)$ & \\
\hline pMI & 226 & 103 (45.6\%) & $123(54.4 \%)$ & \\
\hline PTNM stage & & & & $0.00 I^{*}$ \\
\hline 1 & 89 & $18(22.2 \%)$ & 71 (70.8\%) & \\
\hline ॥ & 81 & 31 (38.3\%) & $50(64.7 \%)$ & \\
\hline III & 143 & $50(35.0 \%)$ & $93(65.0 \%)$ & \\
\hline IV & 225 & 102 (45.3\%) & $123(54.7 \%)$ & \\
\hline
\end{tabular}

Note: *Statistically significant at $P<0.01$.

Abbreviations: $\mathrm{Ln}-\gamma 2$, laminin gamma2; NSCLC, non-small-cell lung cancer; $S$, squamous cell carcinoma; A, adenocarcinoma; $\mathrm{pT}$, pathological tumor; $\mathrm{pN}$, pathological node; pM, pathological metastasis; pTNM, pathological tumor-node-metastasis. 
up to 4 years after diagnosis were included, and 39 patients had been lost to follow-up when the analysis proceeded. Those who were lost to follow-up or died of nontumor causes were regarded as censored data. Peripheral blood samples were collected from each individual at the time of diagnosis and stored in aliquots at $-80^{\circ} \mathrm{C}$. Subjects who had received preoperative adjuvant radio-, chemo, or molecular targeted therapies at enrollment were excluded, as previously described by Teng et al. ${ }^{26}$ This study program, together with the informed consent, was authorized by the Ethics Committee of Beijing Chest Hospital, Capital Medical University. Written informed consent was collected from all individual participants.

\section{Enzyme-linked immunosorbent assay}

Quantitative sandwich enzyme-linked immunosorbent assay (USCN Life Science, Houston, TX, USA) was used to determine the serum content of $\operatorname{Ln}-\gamma 2$ in accordance with the manufacturer's specifications. In brief, $100 \mu \mathrm{L}$ aliquots of diluted sera were incubated in precoated microplates at $37^{\circ} \mathrm{C}$ for 2 hours. Discarding the solution, moderately diluted Ln- $\gamma 2$-detecting antibodies were loaded onto the plates at $37^{\circ} \mathrm{C}$ for 1 hour. After three washes, horseradish peroxidaseconjugated secondary antibody reaction was performed at $37^{\circ} \mathrm{C}$ for 30 minutes. Five more washes later, aliquots of $3,3^{\prime}, 5,5^{\prime}$-tetramethylbenzidine substrate were pipetted into each well and incubated at $37^{\circ} \mathrm{C}$ for 15 minutes under light protection. Sulfuric acid (2 M) solution was finally added to stop the reactions, with the resulting absorbances being determined using a microplate reader (BioTek, Winooski, VT, USA) at $450 \mathrm{~nm}$. The experiment was independently performed three times with each sample in duplicate.

\section{Statistical analysis}

Data normality was checked using the Kolmogorov-Smirnov test. For abnormal distribution, the Wilcoxon rank-sum test was applied to compare the variations between the tumor and control groups, with the serum contents of Ln- $\gamma 2$ in these groups being expressed as medians and ranges. The cutoff value of circulating Ln- $\gamma 2$ was designated as the mean +2 standard deviations of the normal group. For the correlations with serum Ln- $\gamma 2$, clinical variables were categorized and further analyzed by the chi-square test. Overall survival (OS) was counted from the date of diagnosis to the date of cancer death or last follow-up. Survival status was analyzed using the Kaplan-Meier method and compared with the log-rank test. Univariate and multivariate Cox proportional-hazards models were used to predict the influences of the candidate variables in the prognosis of NSCLC. Data analyses were performed with SPSS 19.0, and statistical significance was set as $P<0.05$.

\section{Results High circulating Ln- $\gamma 2$ is an early- emerging event during NSCLC progression}

By serological detection in the healthy group, the cutoff level of serum Ln- $\gamma 2$ in this study was determined as described in the "Materials and methods" section, with the value of 1,568.93 pg/mL (mean: $564.25 \mathrm{pg} / \mathrm{mL}$; standard deviation: $502.34 \mathrm{pg} / \mathrm{mL}$ ). Prominent increases were observed both in terms of the serum content and the positive status of Ln- $\gamma 2$ in the NSCLC group (median: $1,153.30 \mathrm{pg} / \mathrm{mL}$; range: $78.08-4,385.84 \mathrm{pg} / \mathrm{mL}$ ) compared with the values in the normal controls (median: $389.37 \mathrm{pg} / \mathrm{mL}$; range: 0-1,962.60 pg/mL; $P<0.01$; Figure 1A and B), implying its association with certain tumor events. To assess its possible significance in early tumor diagnosis, further comparison was carried out based on the clinical staging of the patients. As viewed in Figure 1C, both the median and the ranges of serum Ln- $\gamma 2$ in stage I patients (median: $872.56 \mathrm{pg} / \mathrm{mL}$, range: $78.08-3,478.80 \mathrm{pg} / \mathrm{mL}$ ) were strikingly elevated relative to those of the normal group $(P<0.01)$, with the corresponding values of the advanced group (stages II-IV: median: 1,228.60 pg/mL, range: $82.51-4,385.84 \mathrm{pg} / \mathrm{mL}$ ) being increased even more. Similarly, significant differences between stage I cases and the normal controls were also reflected directly by the positive status of serum Ln- $\gamma 2$ ( $P=0.01$; Figure 1D), indicating that variation of circulating $\operatorname{Ln}-\gamma 2$ might be an early-emerging tumor event during lung cancer progression. Of note, our data also indicated that serum Ln- $\gamma 2$ was associated strongly with smoking status $(P<0.001)$ and male sex $(P<0.001)$, with higher positive status compared to their counterparts (Table 1), suggesting the relevance of increased serum Ln- $\gamma 2$ levels with smoking-induced lung injury.

\section{Circulating $\operatorname{Ln}-\gamma 2$ is associated strongly with the progression of lung cancer}

Tumor-node-metastasis (TNM) classification is generally required for recognizing the degree of tumor progression, hence serum levels of Ln- $\gamma 2$ were sorted and first analyzed according to the $\mathrm{T}$ stage classification of the pulmonary tumors. As a result, serum Ln- $\gamma 2$ levels were found to increase continuously along with the progression of $\mathrm{T}$ stage (median: T1, 976.88 pg/mL; T2, 1,061.80 pg/mL, T3-T4, $1,414.20 \mathrm{pg} / \mathrm{mL} ; P<0.01$; Figure $2 \mathrm{~A}$ ), with the positive status rising in a consistent manner (Figure 2B, Table 1). As the most representative stage of cancer progression, the status of distant metastasis was further evaluated by comparing serum 
A

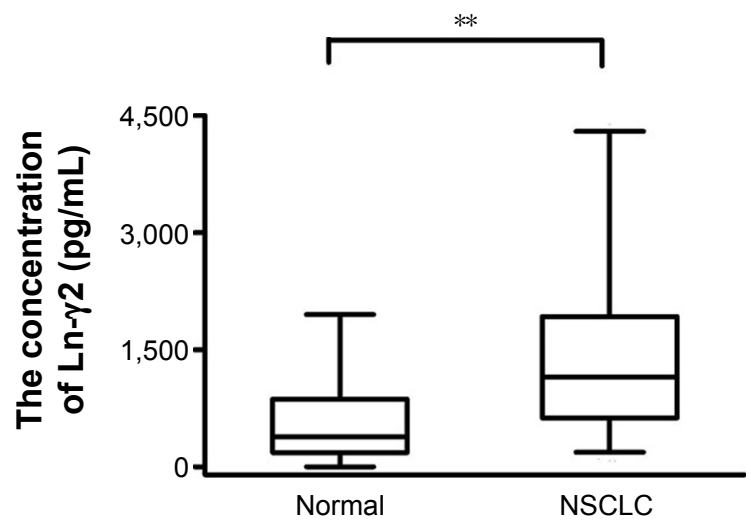

C

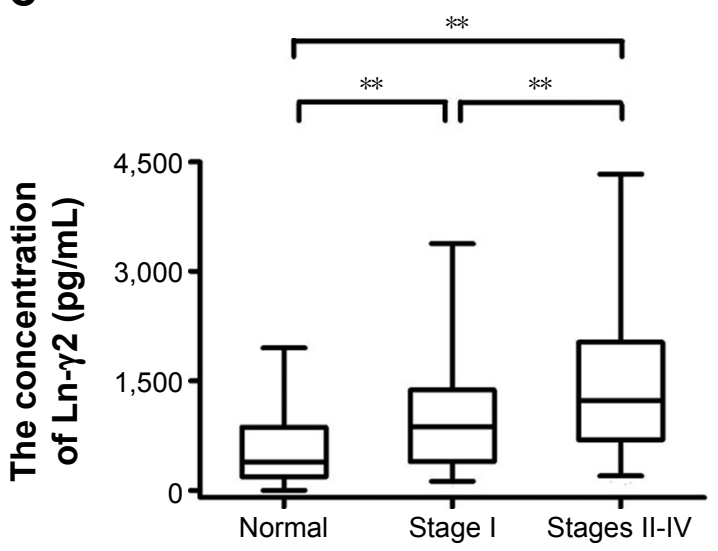

B
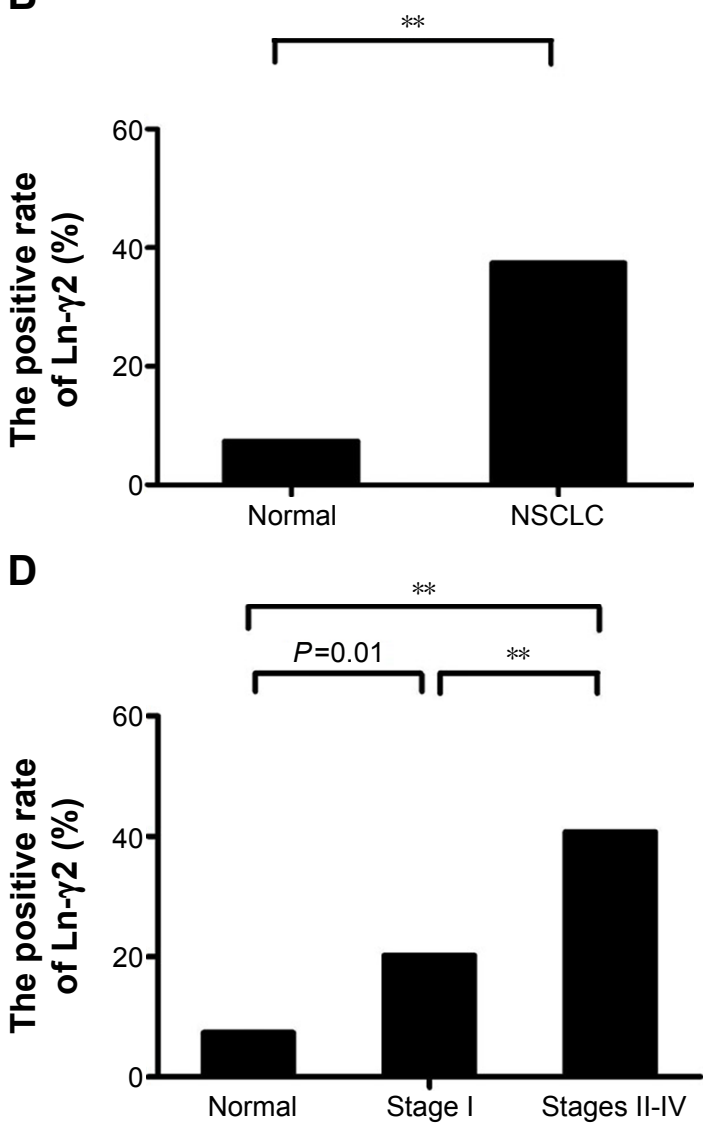

Figure I Variations of serum Ln- $\gamma 2$ levels in NSCLC patients.

Notes: (A) Serum contents of Ln- $\gamma 2$ in the NSCLC and healthy groups. (B) Positive rates of serum Ln- $\gamma 2$ in the NSCLC and healthy groups. (C) Serum contents of Ln- $\gamma 2$ compared between tumor patients of different clinical stages and healthy controls. (D) Positive rates of serum Ln- $\gamma 2$ compared between tumor patients of different clinical stages and healthy controls. Normal, healthy group, $* * P<0.01$.

Abbreviations: Ln- $\gamma 2$, laminin gamma2; NSCLC, non-small-cell lung cancer.

Ln- $\gamma 2$ levels between stage M0 and M1. In comparison with the nonmetastatic (M0 stage, median: $887.73 \mathrm{pg} / \mathrm{mL}$; range: 78.08-3,986.26 $\mathrm{pg} / \mathrm{mL}$ ) patients, the metastatic (M1 stage, median: 1,354.90 pg/mL; range: $87.09-4,385.84 \mathrm{pg} / \mathrm{mL}$ ) cases presented marked increases in terms of both the concentration and positive state of serum Ln- $\gamma 2(P<0.01$; Figure $2 \mathrm{C}$ and D). For regional progression, analysis of lymph node invasion also demonstrated that serum Ln- $\gamma 2$ was more inclined to be positive in higher $\mathrm{N}$ stage tumor patients (Table 1). As a whole, the same tendency of the positive state of serum Ln- $\gamma 2$ was obtained based on the clinical stage classification ( $P=0.001$; Table 1$)$. To avoid interference due to smoking on tumor-derived serum Ln- $\gamma 2$ levels, we next performed the analyses in 213 nonsmokers among the NSCLC cohort. Although some fluctuations emerged in the stages with small sample size, indeed, serum Ln- $\gamma 2$ could still reflect tumor progression based on TNM and clinical stages (Table 2). Therefore, circulating Ln- $\gamma 2$ was proved to vary with tumor stage progression in NSCLC.

\section{High circulating levels of $\mathrm{Ln}-\gamma 2$ predict poor outcome of lung cancer patients}

OS was subsequently investigated in 370 eligible patients who underwent a 4-year follow-up visit by assessing the prognostic potential of circulating Ln- $\gamma 2$ in NSCLC. Kaplan-Meier analysis showed that serum Ln- $\gamma 2$-positive patients survived for dramatically shorter period, with a median survival of 18 months (95\% CI: 12.75-23.25 months), compared to the Ln- $\gamma 2$-negative patients with a 27 -month median survival (95\% CI: 20.01-33.99 months; $P=0.028$; Figure 3A). Due to the early-emerging variation of serum Ln- $\gamma 2$, we wondered whether it could function as an early prognostic indicator of NSCLC. Among the 72 patients in stage I, the negative cases had a prolonged survival (median survival: $>48$ months) compared with their positive counterparts (median survival: 16 months; 95\% CI: 4.60-27.40 months; $P<0.001$; Figure 3B). Of note, in the 298 more advanced cases (stages II-IV), no differences were observed in terms of the survival between serum Ln- $\gamma 2$-positive and -negative groups ( $P=0.830$; Figure $3 \mathrm{C}$ ). 
A
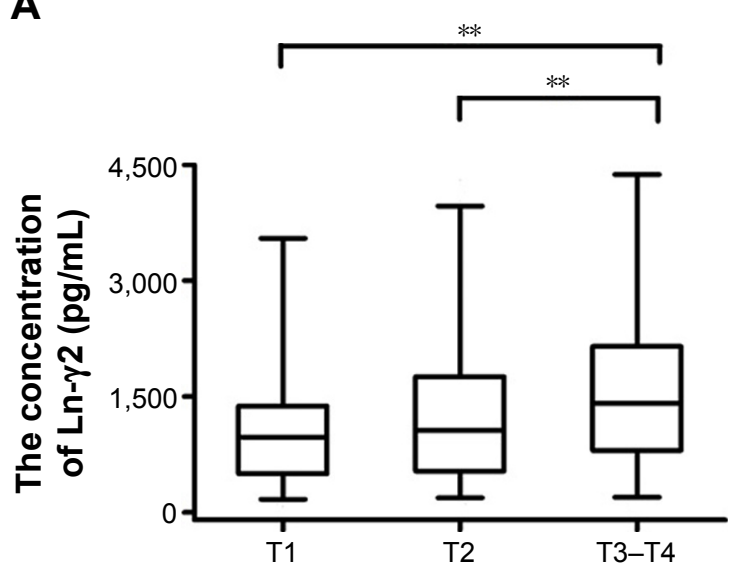

C

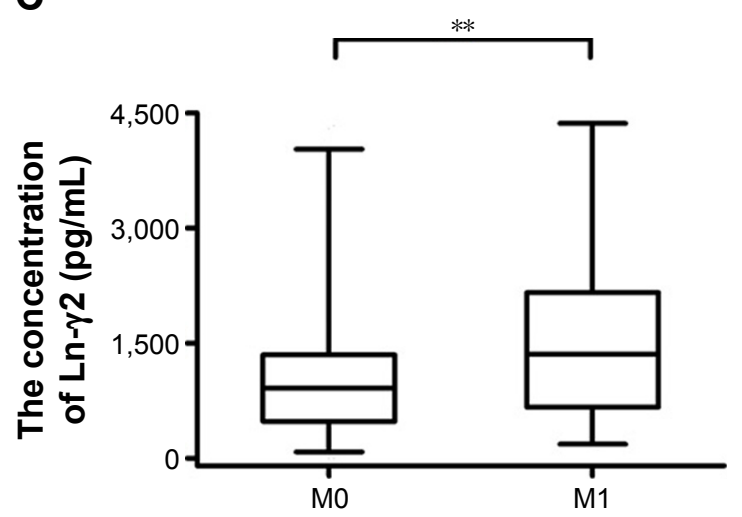

B
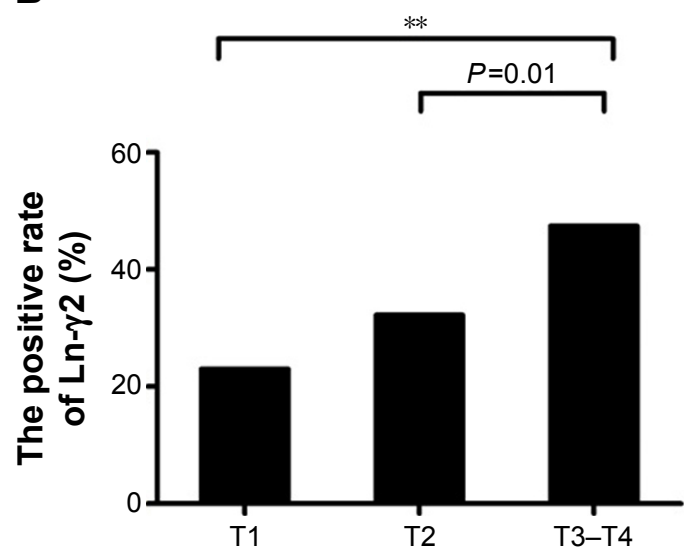

$\mathbf{D}$

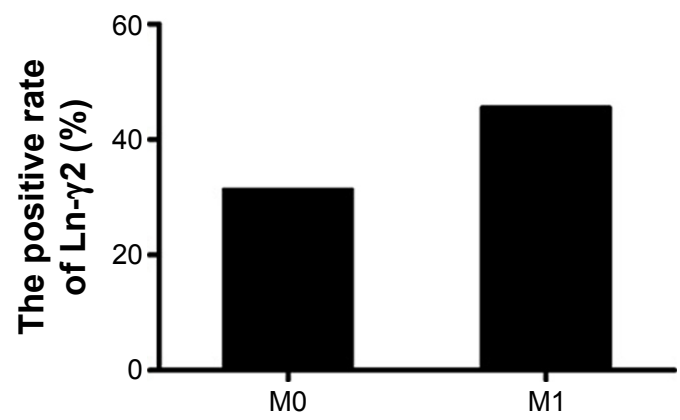

Figure 2 Serum levels of $\mathrm{Ln}-\gamma 2$ altered with lung cancer progression.

Notes: Serum Ln- $\gamma 2$ concentrations (A) and positive rates (B) varied with T stage progression of NSCLC. Serum Ln- $\gamma 2$ concentrations (C) and positive rates (D) increased with $M$ stage progression of NSCLC. $* * P<0.01$.

Abbreviations: Ln- $\gamma 2$, laminin gamma2; NSCLC, non-small-cell lung cancer; T, tumor; M, metastasis.

\section{Circulating $L n-\gamma 2$ is predictive for the prognosis of early-stage NSCLC}

To explore the early prognostic significance of serum Ln- $\gamma 2$ in NSCLC, we further analyzed the survival status on the basis of TNM stages. On the aspect of T stage classification, among the $67 \mathrm{~T} 1$ stage cases, serum Ln- $\gamma 2$-positive patients had much poorer survival (median survival: 17 months, 95\% CI: 11.92-22.08 months) than the Ln- $\gamma 2$-negative group (median survival: $>48$ months; $P=0.001$; Figure 4A). However, in the remaining 303 patients of advanced $\mathrm{T}$ stages (T2-T4), survival analysis showed no significant differences $(P=0.575$; Figure 4B). For the 148 patients of stage N0, the Ln- $\gamma 2$-negative group showed a significantly better survival (median survival: $>48$ months) than the Ln- $\gamma 2$-positive individuals (median survival: 36 months, $P=0.038$; Figure 4C). In the remaining stages of N1-N3, survival curves between the two groups nearly overlapped ( $P=0.669$; Figure 4D). In the context of the 225 non-distant metastatic (M0 stage) cases, the negative patients lived much longer (median survival: $>48$ months) than the positive cases (median survival: 36 months, 95\% CI: 23.29-48.71 months; $P=0.058$; Figure 4E). In the M1 stage cases, as expected, no correlation was found between the positive or negative attribute of serum Ln- $\gamma 2$ and $\mathrm{OS}(P=0.849$; Figure 4F). As for the nonsmoking cohort, similar survival data were observed, which underline the early prognostic importance of serum Ln- $\gamma 2$ (Figure S1).

Cox analysis was further applied to evaluate the prognostic effects of the multiple variables listed in Table 1. In the univariate model, parameters including serum Ln- $\gamma 2(P<0.05)$, TNM status $(P<0.01)$, pathologic type $(P<0.01)$, age $(P<0.05)$, and sex $(P<0.01)$, but not smoking status $(P<0.632)$, were significantly correlated with OS in total cases $(P<0.05$ or $P<0.01$; Table 3$)$. In the multivariate analysis, however, only serum Ln- $\gamma 2(P=0.007)$ and the $\mathrm{N}$ and $\mathrm{M}$ statuses $(P<0.001)$ were found to have independent prognostic significance on tumor survival (Table 3 ). In addition, we also performed multivariate analysis for the more advanced cases (stages II-IV). Notably, 
Table 2 Associations between clinicopathological characteristics and serum Ln- $\gamma 2$ status in 213 nonsmoking tumor patients

\begin{tabular}{|c|c|c|c|c|}
\hline Variables & $\begin{array}{l}\text { No of } \\
\text { cases }\end{array}$ & Ln- $\gamma 2$ positive & Ln- $\gamma 2$ negative & $P$-value \\
\hline Total cases & 213 & $56(26.3 \%)$ & 157 (73.7\%) & \\
\hline Age (years) & & & & 0.412 \\
\hline$\leq 60$ & 124 & $30(24.2 \%)$ & $94(75.8 \%)$ & \\
\hline$>60$ & 89 & $26(29.2 \%)$ & $63(70.8 \%)$ & \\
\hline Sex & & & & 0.107 \\
\hline Male & 62 & 21 (33.9\%) & $4 \mid(66.1 \%)$ & \\
\hline Female & $15 \mid$ & 35 (23.2\%) & $116(76.8 \%)$ & \\
\hline \multicolumn{2}{|c|}{ Pathologic type } & & & 0.997 \\
\hline S & 38 & $10(26.3 \%)$ & $28(73.7 \%)$ & \\
\hline$A$ & 175 & $46(26.3 \%)$ & 129 (73.7\%) & \\
\hline \multicolumn{2}{|c|}{ Differentiation } & & & 0.886 \\
\hline Poor & 84 & $21(25.0 \%)$ & $63(75.0 \%)$ & \\
\hline Moderate & 116 & 32 (27.6\%) & $84(72.4 \%)$ & \\
\hline Well & 13 & $3(23.1 \%)$ & $10(76.9 \%)$ & \\
\hline PT status & & & & 0.074 \\
\hline PTI & 43 & $6(14.0 \%)$ & $37(86.0 \%)$ & \\
\hline pT2 & 84 & $20(23.8 \%)$ & $64(76.2 \%)$ & \\
\hline PT3 & 14 & $5(35.7 \%)$ & $9(64.3 \%)$ & \\
\hline $\mathrm{pT} 4$ & 72 & $25(34.7 \%)$ & 47 (65.3\%) & \\
\hline $\mathrm{pN}$ status & & & & 0.206 \\
\hline pNo & 68 & 14 (20.6\%) & $54(79.4 \%)$ & \\
\hline $\mathrm{pNI}$ & 16 & $4(25.0 \%)$ & $12(75.0 \%)$ & \\
\hline $\mathrm{pN} 2$ & 75 & $18(24.0 \%)$ & 57 (76.0\%) & \\
\hline $\mathrm{pN} 3$ & 54 & $20(37.0 \%)$ & $34(63.0 \%)$ & \\
\hline pM status & & & & $0.006^{* *}$ \\
\hline pMO & 106 & $19(17.9 \%)$ & $87(82.1 \%)$ & \\
\hline pMI & 107 & 37 (34.6\%) & 70 (65.4\%) & \\
\hline PTNM stage & & & & $0.029 *$ \\
\hline 1 & 29 & $3(10.3 \%)$ & $26(89.7 \%)$ & \\
\hline II & 25 & $6(24.0 \%)$ & $19(76.0 \%)$ & \\
\hline III & 52 & $10(19.2 \%)$ & $42(80.8 \%)$ & \\
\hline IV & 107 & 37 (34.6\%) & 70 (65.4\%) & \\
\hline
\end{tabular}

Notes: $* p<0.01$. $* * P<0.05$

Abbreviations: $\operatorname{Ln}-\gamma 2$, laminin gamma2; $\mathrm{S}$, squamous cell carcinoma; A, adenocarcinoma; $\mathrm{pT}$, pathological tumor; $\mathrm{pN}$, pathological node; $\mathrm{pM}$, pathological metastasis; pTNM, pathological tumor-node-metastasis.

only the statuses of lymph node $(\mathrm{N}: P<0.001)$ and distant metastasis $(\mathrm{M} ; P=0.005)$ were significantly associated with OS in the 298 advanced cases, and no statistical significance was noticed on serum Ln- $\gamma 2$ level ( $P=0.234$, Table 4$)$. This analysis further consolidated the finding that serum Ln- $\gamma 2$ is closely associated with the prognosis of early-stage NSCLC, rather than that of advanced cases.

\section{Discussion}

As far as is known, Ln- $\gamma 2$ subunit can be proteolytically processed at the amino-terminal region by certain proteinases, such as matrix metalloprotease-2 (MMP2) ${ }^{27}$ or membranetype MMP (MT1-MMP), ${ }^{28}$ releasing a soluble fragment $(\mathrm{G} 2 \mathrm{~F})$, and thus activating the cell migratory and invasive functions of the Ln-332 trimer. Considering the activating process and the resulting functions of Ln-332, our study focused on the cleaved soluble component of $\operatorname{Ln}-\gamma 2$, aiming to explore the prognostic potential of circulating Ln- $\gamma 2$ in lung cancer treatment. The analytic results delineated a strong correlation between serum $\mathrm{Ln}-\gamma 2$ and tumor stage progression. More importantly, further survival and Cox analyses showed consistent prognostic significances of serum Ln- $\gamma 2$ in each early-stage tumor group, rather than the corresponding advanced groups, suggesting its importance as an effective prognostic indicator of early-stage NSCLC. To our knowledge, this is the first study to address the early prognostic significance of Ln- $\gamma 2$ from the aspect of tumor serology.

Our data on smoking history, commonly known as an induction factor of chronic lung injury, showed a significant association with increasing levels of serum Ln- $\gamma 2$ (Table 1), which was previously considered an early manifestation of alveolar epithelial damage. ${ }^{29}$ Therefore, not only the tumor itself, but also smoking behavior and even other adverse factors, can lead to increased production of serum Ln- $\gamma 2$. However, as demonstrated in this study, regardless of whether a patient smoked or not, serum Ln- $\gamma 2$ levels could have prognostic significance for early-stage NSCLC cases (Figures 4 and S1). From another point of view, smoking-induced elevation of serum Ln- $\gamma 2$, which could further promote migration and invasion of tumor cells, ${ }^{24}$ might somewhat account for the damaging effect of smoking during lung cancer progression.

In this study, we primarily claim the variation of circulating Ln- $\gamma 2$ as an early-emerging prognostic event in NSCLC, not only for stage I patients, but also for early TNM-staged cases, including stages T1, N0, and M0. Representatively, in the small-sized tumor group of stage I (T1-T2aN0M0), no visible lymph node invasion or distant metastasis was detected, while the statistical significance of serum Ln- $\gamma 2$ on early prognosis was the most prominent $(P<0.001$; Figure 3B) among early-stage classifications (T1, $P=0.001$; N0, $P=0.038$; M0, $P=0.058$; Figure 4). In our opinion, this should be attributed to the complex composition of the cases in each early-stage group. In contrast, for the more advanced stages, no predictive value was observed on survival, suggesting that Ln- $\gamma 2$ expression might play an important role in the initiation of regional invasion and metastasis. Further multivariate analyses in all-stage and advanced cases (stages II-IV) verified the speculation that serum Ln- $\gamma 2$ levels are associated preferentially with the prognosis of early-stage NSCLC (Tables 3 and 4). These findings provided a novel insight on the prognostic use of Ln- $\gamma 2$ in cancer therapy and were theoretically in accordance with previous assertions, which regarded Ln- $\gamma 2$ expression as an early part of tumor progression with the increasing potential of invasiveness. ${ }^{21,30}$ Moreover, from the morphological view of tumor invasion, uncoupled deposition 

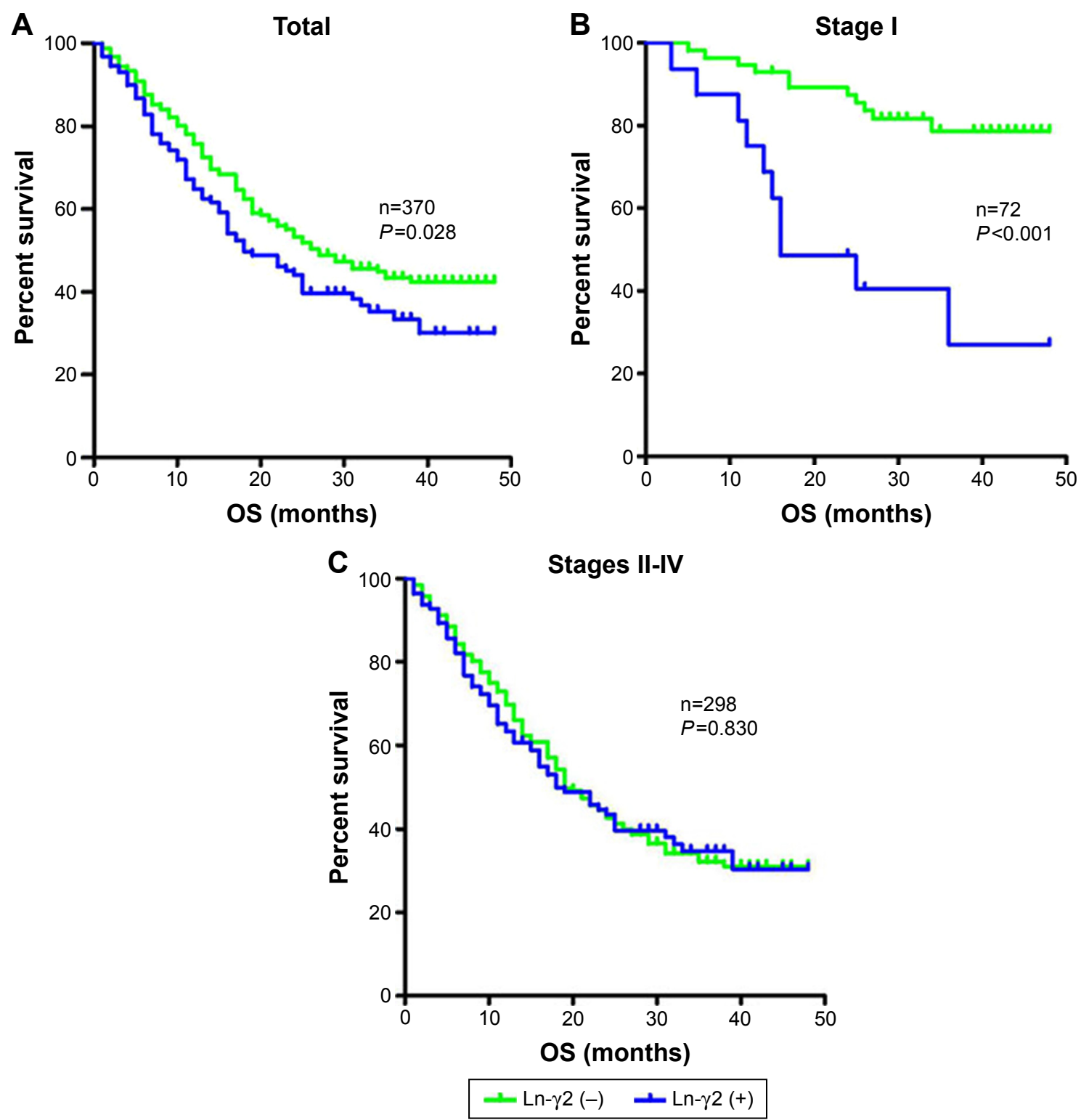

Figure 3 Overall survival status of serum Ln- $\gamma 2$-classified NSCLC patients.

Notes: (A) In total eligible cases, the 4-year OS of serum $L n-\gamma 2$-positive patients was $29.6 \%$, while that of $\mathrm{Ln}$ - $\gamma 2$-negative cases was $42.4 \%$ ( $n=370$, $P=0.028$ ). (B) As for the stage I cases, the OS was $26.8 \%$ for $L n$ - $\gamma 2$-positive patients, but $78.3 \%$ for $L n-\gamma 2$-negative cases $(n=72, P<0.00 I)$. (C) For the more advanced (stages II-IV) cases, analysis showed no differences between the positive $(30.8 \%)$ and negative cases $(31.4 \%)$ on the OS status $(n=298, P=0.830)$.

Abbreviations: Ln- $\gamma 2$, laminin gamma2; NSCLC, non-small-cell lung cancer; OS, overall survival.

or secretion of gamma2 monomer frequently occurred at the frontier of invading tumors, ${ }^{15}$ and intensity of tumor budding was found to be definitely associated with $\mathrm{Ln}-\gamma 2$ expression, indicating its importance during the establishment of an invasive phenotype. ${ }^{31}$ To date, the molecular mechanisms of Ln- $\gamma 2$ expression are still limited, among which $W n t / \beta$-catenin signaling functions predominantly as an upstream regulatory pathway. ${ }^{12,32}$ Numerous clinical studies have confirmed the $\beta$-catenin pathway to be closely involved in early-stage tumor invasion and progression, ${ }^{33,34}$ during which enhanced $\beta$-catenin accumulation is usually accompanied with the disruption of E-cadherin $/ \beta$-catenin complex, ${ }^{35}$ a membrane component crucial for intercellular adhesion. Such molecular changes might causally pave the way for the promotion of Ln- $\gamma 2$ expression. Interestingly, previous evidence also proved that the tumor suppressor Smad4 could mediate positive targeted regulation of $\mathrm{Ln}-\gamma 2$ and the other two subunits of the heterotrimer, ${ }^{36}$ which appeared incompatible with the proinvasive activity of Ln- $\gamma 2$ and suggested complicated regulations of Ln- $\gamma 2$ expression. However, it was actually reasonable in consideration of the adhesive properties of the BM component, Ln-332.

As an early-emerging tumor event, Ln- $\gamma 2$ overexpression is not only correlated with cell migration and invasion 

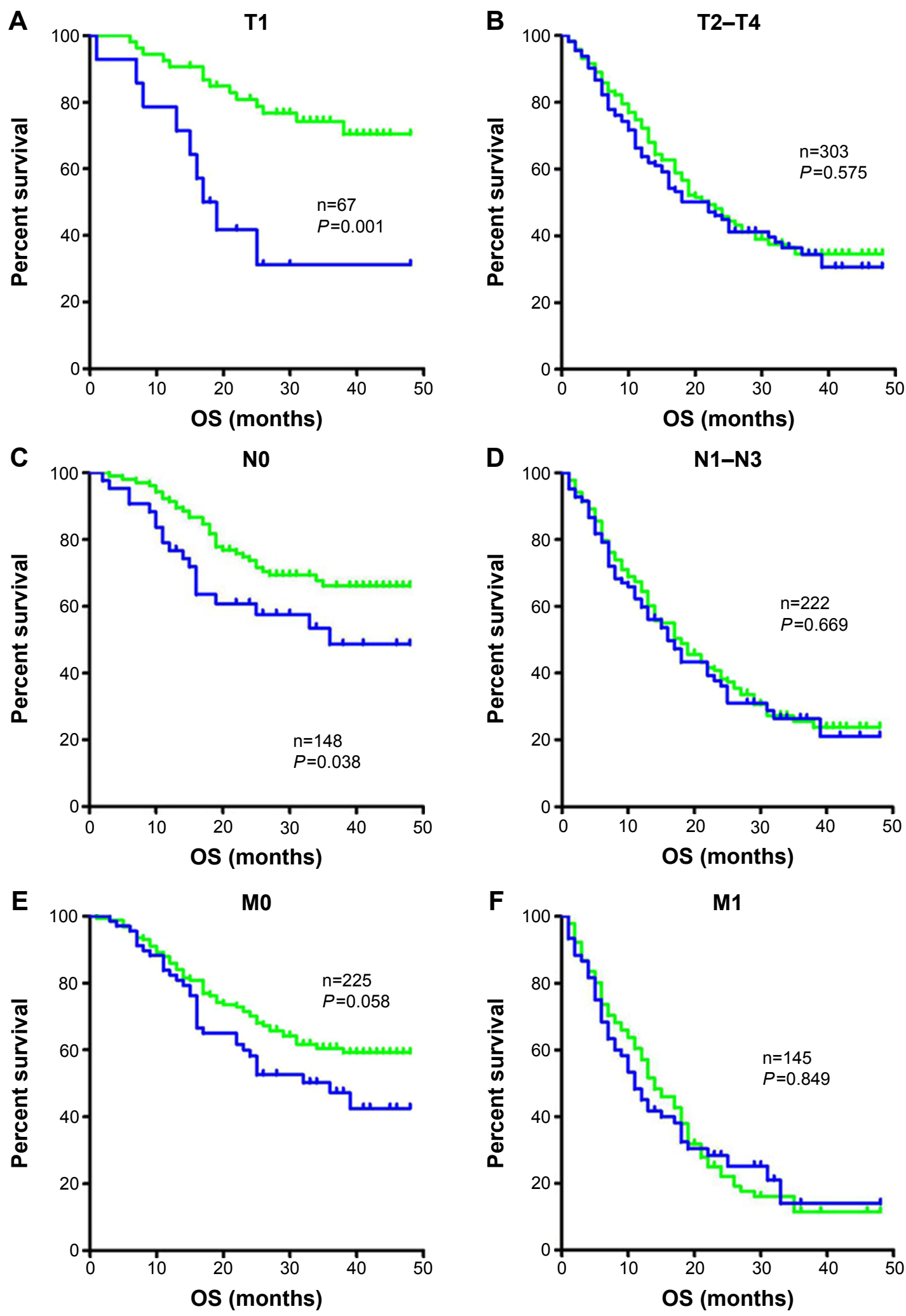

$\perp \operatorname{Ln}-\gamma 2(-) \quad L \quad \operatorname{Ln}-\gamma 2(+)$

Figure 4 Prognostic significance of serum Ln- $\gamma 2$ in early TNM stages of NSCLC.

Notes: (A) In stage TI, the 4-year OS was $31.7 \%$ for serum $\mathrm{Ln}$ - $\gamma 2$-positive cases, but $73.6 \%$ for the negative cases ( $\mathrm{n}=67, \mathrm{P}=0.00 \mathrm{I})$. (B) In advanced stages of T2-T4, analysis showed no differences between the positive (29.8\%) and negative cases $(34.3 \%)$ on comparing the OS rate $(n=303, P=0.575)$. (C) In stage N0, the OS of $L n-\gamma 2$-positive cases was $48.8 \%$, while that of $L n-\gamma 2$-negative cases was $68.6 \%(n=148, P=0.038)$. (D) In advanced stages of $\mathrm{NI}-\mathrm{N} 3$, no significant differences were observed between the positive (21.5\%) and negative cases (23.4\%) on the OS comparison $(n=222, P=0.669)$. (E) In stage M0, the OS was $41.9 \%$ for $L n-\gamma 2$-positive cases and $59.4 \%$ for the negative cases ( $n=225, P=0.058)$. (F) In metastatic stage $M I$, no differences were observed between the positive $(I 3.1 \%)$ and negative cases $(I I .7 \%)$ on the OS rate $(n=I 45, P=0.849)$.

Abbreviations: Ln- $\gamma 2$, laminin gamma2; TNM, tumor-node-metastasis; NSCLC, non-small-cell lung cancer; OS, overall survival; T, tumor; N, node; M, metastasis. 
Table 3 Cox regression analyses on the overall survival of NSCLC patients $(n=370)$

\begin{tabular}{|c|c|c|c|c|}
\hline \multirow[t]{2}{*}{ Variables } & \multicolumn{2}{|l|}{ Univariate analysis } & \multicolumn{2}{|l|}{ Multivariate analysis } \\
\hline & Risk ratio $(95 \% \mathrm{Cl})$ & $P$-value & Risk ratio (95\% Cl) & $P$-value \\
\hline Age (>60 years) & $1.005-1.725$ & $0.046^{*}$ & $0.858-1.864$ & 0.235 \\
\hline Sex (female) & $1.202-2.113$ & $0.001 * *$ & $0.846-3.136$ & 0.145 \\
\hline Smoking status (yes) & $0.708-1.234$ & 0.632 & $0.633-2.133$ & 0.629 \\
\hline Pathologic type (S) & $0.504-0.884$ & $0.005^{* *}$ & $0.688-1.630$ & 0.796 \\
\hline Differentiation ( $M$ and $W$ ) & $0.5 \mathrm{II}-1.075$ & 0.114 & $0.512-1.002$ & 0.510 \\
\hline PT status (T3-T4) & $1.802-3.127$ & $0.000 * *$ & $0.784-1.992$ & 0.349 \\
\hline pN status (NI-N3) & $2.127-4.010$ & $0.000 * *$ & $1.664-3.903$ & $0.000 * *$ \\
\hline pM status (MI) & $2.607-4.556$ & $0.000 * *$ & $1.562-3.900$ & $0.000 * *$ \\
\hline Serum Ln- $\gamma 2$ (positive) & $1.021-1.788$ & $0.035^{*}$ & I.168-2.649 & $0.007^{* *}$ \\
\hline
\end{tabular}

Notes: $* P<0.05$. $* * P<0.01$.

Abbreviations: $\mathrm{Cl}$, confidence interval; NSCLC, non-small-cell lung cancer; $\mathrm{M}$ and W, moderate and well differentiated; Ln- $\gamma 2$, laminin gamma2; S, squamous cell carcinoma; PT, pathological tumor; pN, pathological node; pM, pathological metastasis; T, tumor; N, node; $M$, metastasis.

but also with cell viability and infiltrative growth. ${ }^{10,14}$ The latter capacities could be corroborated, in this study, by the continuous increase of serum $\mathrm{Ln}-\gamma 2$ during the progression of tumor size (Figure 2A and Table 1). A recent report documented that increased expression of Ln-332, especially the gamma2 monomer, could promote aberrant proliferation via integrin- $\alpha 6 \beta 4$, one of its receptors, and the downstream ERK activation. ${ }^{37}$ Furthermore, several repeats of the epidermal growth factor (EGF)-like domain located in the short arm of Ln- $\gamma 2$, as potential ligands of the cell surface EGF receptors, were previously proposed to initiate activation of signaling pathways, such as the MAPK/ERK pathway after cleavage by certain proteinases, and thus to modulate cell migratory and proliferative capacities. ${ }^{13,38}$ Exposure of these EGF-like domains by proteolysis raised an alternative approach independent of integrin-mediated signaling pathway, suggesting the diversity of signaling mechanisms between epithelial cells and surrounding extracellular matrix components. Not surprisingly, series of clinicopathological results have confirmed the internal correlations between Ln- $\gamma 2$ and EGF receptor

Table 4 Multivariate analysis on the overall survival of stages II-IV NSCLC patients $(\mathrm{n}=298)$

\begin{tabular}{lll}
\hline Variables & \multicolumn{2}{l}{ Multivariate analysis } \\
\cline { 2 - 3 } & Risk ratio $(\mathbf{9 5 \%} \mathbf{~ C l )}$ & P-value \\
\hline Smoking status (yes) & $0.422-1.137$ & 0.146 \\
Pathologic type (S) & $0.833-2.255$ & 0.215 \\
Differentiation (M and W) & $0.493-1.180$ & 0.224 \\
PT status (T3-T4) & $0.813-2.067$ & 0.276 \\
PN status (NI-N3) & $1.623-5.44 I$ & $0.000^{*}$ \\
pM status (MI) & $1.22 I-3.078$ & $0.005^{*}$ \\
Serum Ln- $\gamma 2$ (positive) & $0.86 I-2.294$ & 0.234 \\
\hline
\end{tabular}

Notes: $\mathrm{S}$, squamous cell carcinoma. ${ }^{*} P<0.01$.

Abbreviations: NSCLC, non-small-cell lung cancer; $M$ and $W$, moderate and well differentiated; $\mathrm{Ln}-\gamma 2$, laminin gamma2; Cl, confidence interval; $\mathrm{pT}$, pathological tumor; pN, pathological node; pM, pathological metastasis; $\mathrm{T}$, tumor; $\mathrm{N}$, node; $\mathrm{M}$, metastasis. expression in vivo, ${ }^{39,40}$ indicating their coexpression with a more efficient predictive value for cancer prognosis.

In this study, we made an effort to determine the importance of circulating Ln- $\gamma 2$ as an early prognostic indicator of NSCLC. Despite a relatively large sample size, some limitations of the project, such as single-center and retrospective designs, might cause selection bias. Furthermore, pretreatment serological analysis might conceal the possible capacities of serum Ln- $\gamma 2$ on the monitoring of the therapeutic response and early recurrence of lung cancer. In the future, a multicenter prospective study with consecutive collection of posttreatment sera until relapse may be more contributive to widely exploring the potentials of serum Ln- $\gamma 2$ on early prognosis, response evaluation, and early recurrence prediction of lung cancer.

\section{Conclusion}

In summary, our findings indicate that increase of circulating Ln- $\gamma 2$ is an early-emerging event in NSCLC and may be a helpful indicator for early diagnosis of lung cancer. Importantly, circulating Ln- $\gamma 2$ is significantly associated with the prognosis of NSCLC, especially for early-stage cases. Thus, circulating Ln- $\gamma 2$ might be a convenient and applicable prognostic biomarker for early-stage NSCLC. Further research on the mechanism of Ln- $\gamma 2$ signaling might provide a promising target for cancer therapy.

\section{Acknowledgments}

We are grateful to the staff of Beijing Chest Hospital Sample Bank for help in serum collection and management. This work was supported by the Natural Science Foundation of China (grant no 61431019), Beijing Natural Science Foundation (grant no 7154189), and the Capital Health Research and Development of Special Fund (grant no 2014-2-1041). 


\section{Disclosure}

The authors report no conflicts of interest in this work.

\section{References}

1. Youlden DR, Cramb SM, Baade PD. The international epidemiology of lung cancer: geographical distribution and secular trends. J Thorac Oncol. 2008;3(8):819-831.

2. Fidler IJ. The pathogenesis of cancer metastasis: the 'seed and soil' hypothesis revisited. Nat Rev Cancer. 2003;3(6):453-458.

3. Mori T, Ono K, Kariya Y, Ogawa T, Higashi S, Miyazaki K. Laminin3B11, a novel vascular-type laminin capable of inducing prominent lamellipodial protrusions in microvascular endothelial cells. $J$ Biol Chem. 2010;285(45):35068-35078.

4. Chung H, Suh EK, Han IO, Oh ES. Keratinocyte-derived laminin-332 promotes adhesion and migration in melanocytes and melanoma. $\mathrm{J} \mathrm{Biol}$ Chem. 2011;286(15):13438-13447.

5. Kiritsi D, Has C, Bruckner-Tuderman L. Laminin 332 in junctional epidermolysis bullosa. Cell Adh Migr. 2013;7(1):135-141.

6. Kim BG, An HJ, Kang S, et al. Laminin-332-rich tumor microenvironment for tumor invasion in the interface zone of breast cancer. Am J Pathol. 2011;178(1):373-381.

7. Drake JM, Barnes JM, Madsen JM, Domann FE, Stipp CS, Henry MD. ZEB1 coordinately regulates laminin-332 and \{beta $\} 4$ integrin expression altering the invasive phenotype of prostate cancer cells. $J$ Biol Chem. 2010;285(44):33940-33948.

8. Pyke C, Rømer J, Kallunki P, et al. The gamma 2 chain of kalinin/laminin 5 is preferentially expressed in invading malignant cells in human cancers. Am J Pathol. 1994;145(4):782-791.

9. Niki T, Kohno T, Iba S, et al. Frequent co-localization of Cox-2 and laminin-5 gamma2 chain at the invasive front of early-stage lung adenocarcinomas. Am J Pathol. 2002;160(3):1129-1141.

10. Kim BG, Gao MQ, Choi YP, et al. Invasive breast cancer induces laminin-332 upregulation and integrin $\beta 4$ neoexpression in myofibroblasts to confer an anoikis-resistant phenotype during tissue remodeling. Breast Cancer Res. 2012;14(3):R88.

11. Koshikawa N, Moriyama K, Takamura H, et al. Overexpression of laminin gamma2 chain monomer in invading gastric carcinoma cells. Cancer Res. 1999;59(21):5596-5601.

12. Yamamoto H, Kitadai Y, Yamamoto H, et al. Laminin gamma2 mediates Wnt5a-induced invasion of gastric cancer cells. Gastroenterology. 2009;137(1):242-252.

13. Hamasaki H, Koga K, Aoki M, et al. Expression of laminin 5-gamma2 chain in cutaneous squamous cell carcinoma and its role in tumour invasion. Br J Cancer. 2011;105(6):824-832.

14. Tsubota Y, Ogawa T, Oyanagi J, Nagashima Y, Miyazaki K. Expression of laminin gamma 2 chain monomer enhances invasive growth of human carcinoma cells in vivo. Int J Cancer. 2010;127(9):2031-2041.

15. Zboralski D, Warscheid B, Klein-Scory S, et al. Uncoupled responses of Smad4-deficient cancer cells to TNFalpha result in secretion of monomeric laminin-gamma2. Mol Cancer. 2010;9(1):65.

16. Noel JC, Fernandez-Aguilar S, Fayt I, et al. Laminin-5 gamma 2 chain expression in cervical intraepithelial neoplasia and invasive cervical carcinoma. Acta Obstet Gynecol Scand. 2005;84(11): 1119-1123.

17. Degen M, Natarajan E, Barron P, Widlund HR, Rheinwald JG. MAPK/ ERK-dependent translation factor hyperactivation and dysregulated laminin gamma2 expression in oral dysplasia and squamous cell carcinoma. Am J Pathol. 2012;180(6):2462-2478.

18. Shinto E, Tsuda H, Ueno H, et al. Prognostic implication of laminin-5 gamma 2 chain expression in the invasive front of colorectal cancers, disclosed by area-specific four-point tissue microarrays. Lab Invest. 2005;85(2):257-266.

19. Liu W, Tian F, Jiang P, et al. Aberrant expression of laminin gamma2 correlates with poor prognosis and promotes invasion in extrahepatic cholangiocarcinoma. J Surg Res. 2014;186(1):150-156.
20. Masuda R, Kijima H, Imamura N, et al. Laminin-5gamma2 chain expression is associated with tumor cell invasiveness and prognosis of lung squamous cell carcinoma. Biomed Res. 2012;33(5):309-317.

21. Lenander C, Roblick UJ, Habermann JK, Ost A, Tryggvason K, Auer G. Laminin 5 gamma 2 chain expression: a marker of early invasiveness in colorectal adenomas. Mol Pathol. 2003;56(6):342-346.

22. Masaki T, Matsuoka H, Sugiyama M, et al. Laminin-5 gamma2 chain expression as a possible determinant of tumor aggressiveness in T1 colorectal carcinomas. Dig Dis Sci. 2003;48(2):272-278.

23. Katayama M, Sanzen N, Funakoshi A, Sekiguchi K. Laminin gamma2chain fragment in the circulation: a prognostic indicator of epithelial tumor invasion. Cancer Res. 2003;63(1):222-229.

24. Katayama M, Funakoshi A, Sumii T, Sanzen N, Sekiguchi K. Laminin gamma2-chain fragment circulating level increases in patients with metastatic pancreatic ductal cell adenocarcinomas. Cancer Lett. 2005; 225(1):167-176.

25. Kuratomi Y, Sato S, Monji M, et al. Serum concentrations of laminin gamma2 fragments in patients with head and neck squamous cell carcinoma. Head Neck. 2008;30(8):1058-1063.

26. Teng Y, Xu S, Yue W, et al. Serological investigation of the clinical significance of fascin in non-small-cell lung cancer. Lung Cancer. 2013; 82(2):346-352.

27. Giannelli G, Falk-Marzillier J, Schiraldi O, Stetler-Stevenson WG, Quaranta V. Induction of cell migration by matrix metalloprotease-2 cleavage of laminin-5. Science. 1997;277(5323):225-228.

28. Koshikawa N, Giannelli G, Cirulli V, Miyazaki K, Quaranta V. Role of cell surface metalloprotease MT1-MMP in epithelial cell migration over laminin-5. J Cell Biol. 2000;148(3):615-624.

29. Katayama M, Ishizaka A, Sakamoto M, et al. Laminin gamma2 fragments are increased in the circulation of patients with early phase acute lung injury. Intensive Care Med. 2010;36(3):479-486.

30. Lindberg P, Larsson A, Nielsen BS. Expression of plasminogen activator inhibitor-1, urokinase receptor and laminin gamma-2 chain is an early coordinated event in incipient oral squamous cell carcinoma. Int $J$ Cancer. 2006;118(12):2948-2956.

31. Marangon Junior H, Rocha VN, Leite CF, de Aguiar MC, Souza PE, Horta MC. Laminin-5 gamma 2 chain expression is associated with intensity of tumor budding and density of stromal myofibroblasts in oral squamous cell carcinoma. J Oral Pathol Med. 2014;43(3):199-204.

32. Hlubek F, Spaderna S, Jung A, Kirchner T, Brabletz T. Beta-catenin activates a coordinated expression of the proinvasive factors laminin-5 gamma 2 chain and MT1-MMP in colorectal carcinomas. Int J Cancer. 2004;108(2):321-326.

33. Noordhuis MG, Fehrmann RS, Wisman GB, et al. Involvement of the TGF-beta and beta-catenin pathways in pelvic lymph node metastasis in early-stage cervical cancer. Clin Cancer Res. 2011;17(6):1317-1330.

34. Watson AL, Rahrmann EP, Moriarity BS, et al. Canonical Wnt/betacatenin signaling drives human Schwann cell transformation, progression, and tumor maintenance. Cancer Discov. 2013;3(6):674-689.

35. Han J, Kim Y, Kim DY, Na KJ. Alteration in E-cadherin/beta-catenin expression in canine melanotic tumors. Vet Pathol. 2013;50(2):274-280.

36. Zboralski D, Bockmann M, Zapatka M, et al. Divergent mechanisms underlie Smad4-mediated positive regulation of the three genes encoding the basement membrane component laminin-332 (laminin-5). BMC Cancer. 2008;8:215.

37. Vijayakumar S, Dang S, Marinkovich MP, et al. Aberrant expression of laminin-332 promotes cell proliferation and cyst growth in ARPKD. Am J Physiol Renal Physiol. 2014;306(6):F640-F654.

38. Schenk S, Hintermann E, Bilban M, et al. Binding to EGF receptor of a laminin-5 EGF-like fragment liberated during MMP-dependent mammary gland involution. J Cell Biol. 2003;161(1):197-209.

39. Fukai Y, Masuda N, Kato H, et al. Correlation between laminin-5 gamma2 chain and epidermal growth factor receptor expression in esophageal squamous cell carcinomas. Oncology. 2005;69(1):71-80.

40. Sentani K, Matsuda M, Oue N, et al. Clinicopathological significance of MMP-7, laminin gamma2 and EGFR expression at the invasive front of gastric carcinoma. Gastric Cancer. 2014;17(3):412-422. 


\section{Supplementary material}
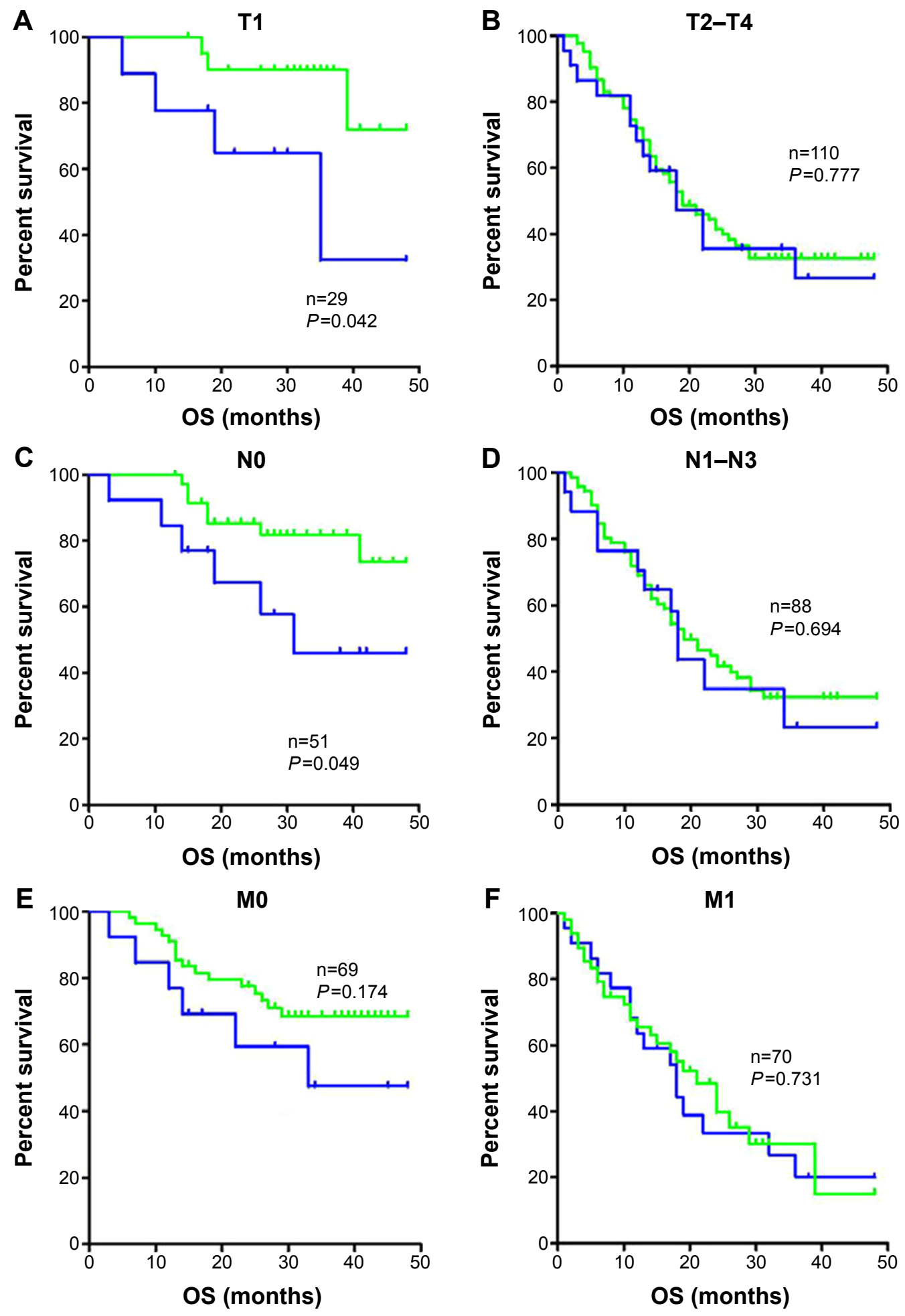

$$
\perp \operatorname{Ln}-\gamma 2(-) \perp \operatorname{Ln}-\gamma 2(+)
$$

Figure SI Prognostic significance of serum Ln- $\gamma 2$ in early TNM stages of non-smoking NSCLC patients $(n=139)$.

Notes: (A, C, and E) In the early stages of TI, N0, and M0, the 4-year overall survivals of serum Ln- $\gamma 2$-positive cases were significantly lower than those of Ln- $\gamma 2$-negative patients; (B, D, and F) while in the more advanced stages of T2-T4, NI-N3, and MI, no significant differences were observed between serum Ln- $\gamma 2$ positive and negative cases on the OS rate.

Abbreviations: Ln- $\gamma 2$, laminin gamma2; TNM, tumor-node-metastasis; NSCLC, non-small-cell lung cancer; OS, overall survival; T, tumor; N, node; M, metastasis. 


\section{Publish your work in this journal}

OncoTargets and Therapy is an international, peer-reviewed, open access journal focusing on the pathological basis of all cancers, potential targets for therapy and treatment protocols employed to improve the management of cancer patients. The journal also focuses on the impact of management programs and new therapeutic agents and protocols on

patient perspectives such as quality of life, adherence and satisfaction. The manuscript management system is completely online and includes a very quick and fair peer-review system, which is all easy to use. Visit http://www.dovepress.com/testimonials.php to read real quotes from published authors.

Submit your manuscript here: http://www.dovepress.com/oncotargets-and-therapy-journal 